\title{
Clinical manifestations in trisomy 9 mosaicism
}

\author{
Ljiljana Pejcic, Tatijana Stankovic, Marija Ratkovic-Jankovic, Karin Vasic, Ivana Nikolic \\ Clinic of Children's Internal Diseases, Clinical Center Niš, Nǐ̌, Serbia. E-mail: ljiljasonja@yahoo.com \\ Received: 24th January 2017, Revised: 5th May 2017, 24th August 2017, Accept: 30th November 2017
}

SUMMARY: Pejcic L, Stankovic T, Ratkovic-Jankovic M, Vasic K, Nikolic I. Clinical manifestations in trisomy 9 mosaicism. Turk J Pediatr 2018; 60: 729734.

Trisomy 9 is a rare chromosome abnormality which can occur in a mosaic or nonmosaic state with similar clinical features. The authors present a male with mosaic trisomy 9 from birth to 6 months of life. Clinical manifestations included growth retardation, facial dysmorphism with marked hemi facial hypoplasia and facial asymmetry, single palmar flexion crease, retro calcaneovalgus feet, atrial septal defect, undescended testes and hypospadia. He had several episodes of seizures and ultrasound examination described severe ventriculomegaly, with poorly differentiated parenchyme. These findings are compared to the other previously described cases of trisomy 9 mosaicism through a review of literature.

Key words: mosaic trisomy 9, facial asymmetry, developmental delay, children.

Trisomy 9 is an uncommon chromosome abnormality which can occur in a mosaic or nonmosaic state. It presents with a distinct clinical picture. Full trisomy 9 is rare in live born infants ${ }^{1}$, but trisomy 9 mosaicism has been reported and is compatible with life. The range of clinical manifestation is variable and has not been well documented, especially the mental status of those children. Patients usually present with similar clinical features including growth retardation, facial features, cardiac, renal, and skeletal abnormalities. ${ }^{2-5}$ Most patients who survive are severely mentally delayed.

\section{Case Report}

We present, with the mother's consent, a male from birth to 6 months of life with mosaic trisomy 9 and typical clinical manifestations: facial and skeletal dysmorphism, various internal organ malformations and suspected psychomotor retardation.

A male infant was admitted to the Neonatal Intensive Care Unit at the age of 3 days with multiple congenital anomalies. He was delivered by Cesarean section in 36/37 week gestation of a 24-year old gravida as a first child. Before and during the first trimester mother had condylomas, which resolved during the fourth month of pregnancy. There were no known other complaints, nor exposure to irradiation or infection. Also we could not be sure that there was no consanguinity, or family history of birth defects or mental delay, because the mother was an adopted child and there were no data about her family. As there was a suspicion of reduced amounts of amniotic fluid and intrauterine growth retardation during the last trimester of pregnancy, delivery was at $36 / 37$ th week by Cesarean section. Birth weight was $1540 \mathrm{~g}$, length $43 \mathrm{~cm}$, occipitofrontal circumference $30 \mathrm{~cm}\left(<3^{\text {rd }}\right.$ centile $\left.32 \mathrm{~cm}\right)$, and Apgar scores were 5 and 7 at 1 and 5 minutes. Additional to short stature and slow growth, the newborn presented with multiple craniofacial and skeletal abnormalities. His facial features were similar to trisomy 13: short palpebral fissures, enophtalmus, bulbous nose, micrognatia, high-arched palate and low-set malformed ears were noted. The child had sloping forehead with narrow bifrontal diameter, facial asymmetry, upslanting palpebral fissures, prominent nasal bridge with short root and prominent lip covering receding lower lip (Fig. 1). He had a single crease in the palm of the hand (Fig. 2). He also had undescended testes and hypospadias. There was limitation of motion of the hips and retro calcaneovalgus feet. The neonate presented with a grade III systolic murmur and echocardiography revealed 

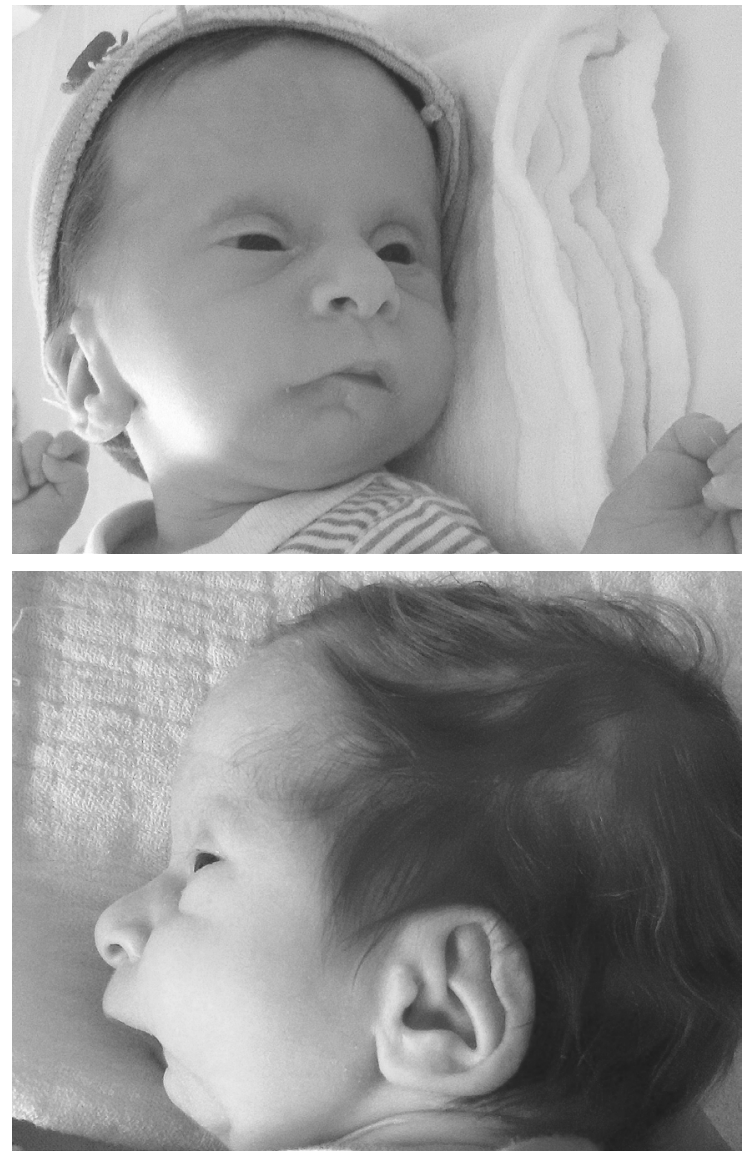

Fig. 1a and 1b. Front and side views of the dysmorphic face and low-set abnormally shaped ears.

a small atrial septal defect. He had a low pitched and decreased Moro reflex. During the early neonatal period, our patient required only diffuse oxygen therapy (FiO2 40\%), without need for mechanical ventilation. He did not have apnea during follow-up. Laboratory investigations (blood and urine analyses) were mostly normal. The red and white cell

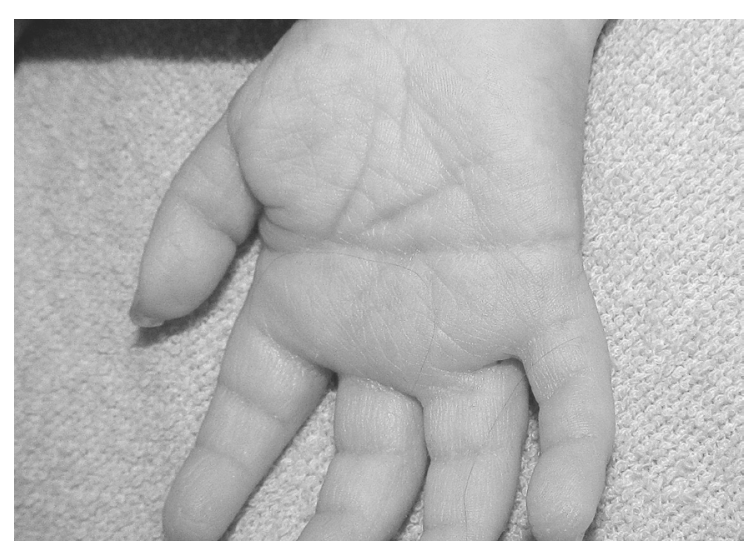

Fig. 2. Single crease in the palm. count were normal from birth onwards, except for mild leucocytosis (20 000 cells $/ \mu \mathrm{l}$ ) with neutrofilia and low platetlet count noted during treatment of late neonatal sepsis. At this time Staphylocccus sp. was isolated from a blood culture. Electrolytes, except for mild hypocalcemia $(1.95 \mathrm{mmol} / \mathrm{L})$ registered during the early neonatal period, acid-base status; liver panel testing were also normal. Karyotype analysis was performed from peripheral blood

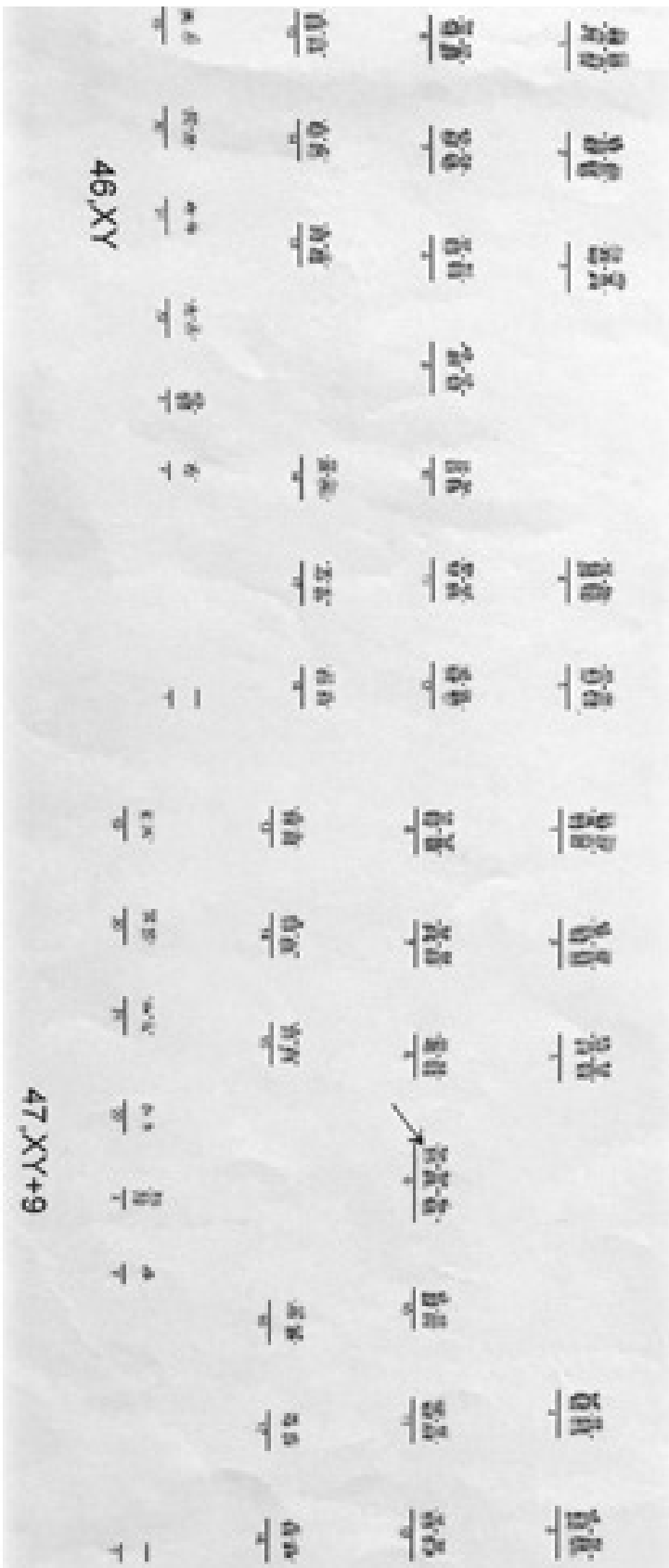

Fig. 3. The karyotype of the patient showing $47, \mathrm{XY},+9$ 3/46,XY 27. 
cell culture and it was 47,XY,+9 3/46,XY 27 (Fig. 3.). His parents' karyotypes were normal. We followed the infant for the first six months of life. The baby gained weight poorly and at the end of 6 months his weight was $3670 \mathrm{~g}$ $\left(<3^{\text {rd }}\right.$ centile $\left.3500 \mathrm{~g}\right)$, head circumference 39 $\mathrm{cm}\left(<3^{\text {rd }}\right.$ centile $\left.41 \mathrm{~cm}\right)$ with marked hemi facial hypoplasia and facial asymmetry - right hemifacial microsomia (Fig. 4). The infant had hypotonia, could not lift his head in prone position, nor was sitting (Fig. 5). According to the Denver Developmental Screening test, at age of 6 months, the patient's gross motor skills, fine motor-adaptive, language and personalsocial skills were at the level of age $0-1$ month, thereby depicting enormous developmental delay. He had already had several episodes of seizures, but antiepileptic medication kept them under control. Cranial ultrasound examination detected severe ventriculomegaly, with poorly differentiated parenchyme. Auditory assessment was performed by means of auditory evoked potentials. Bilateral hypoacusis was detected, with hearing threshold of $65 \mathrm{~dB}$ in the left ear and $40 \mathrm{~dB}$ in the right ear. Ocular examination did not reveal microphthalmus, however mild (grade 1) retinal hemorrhage in the right eye was noted and visual impairment suspected. Genitourinary anomalies were presented as undescended testes and hypospadia (Fig. 6). Ultrasound examination confirmed that the testes were located in the inguinal canals. He developed a urinary tract infection during the course of hospitalization which responded well to antibiotic treatment. Ultrasound examination of the urinary tract revealed hydronephrosis

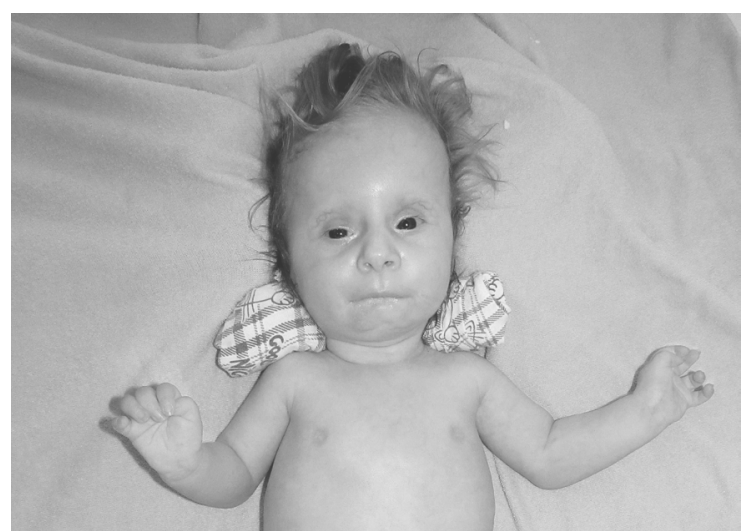

Fig. 4. Marked hemi facial hypoplasia with facial asymmetry.
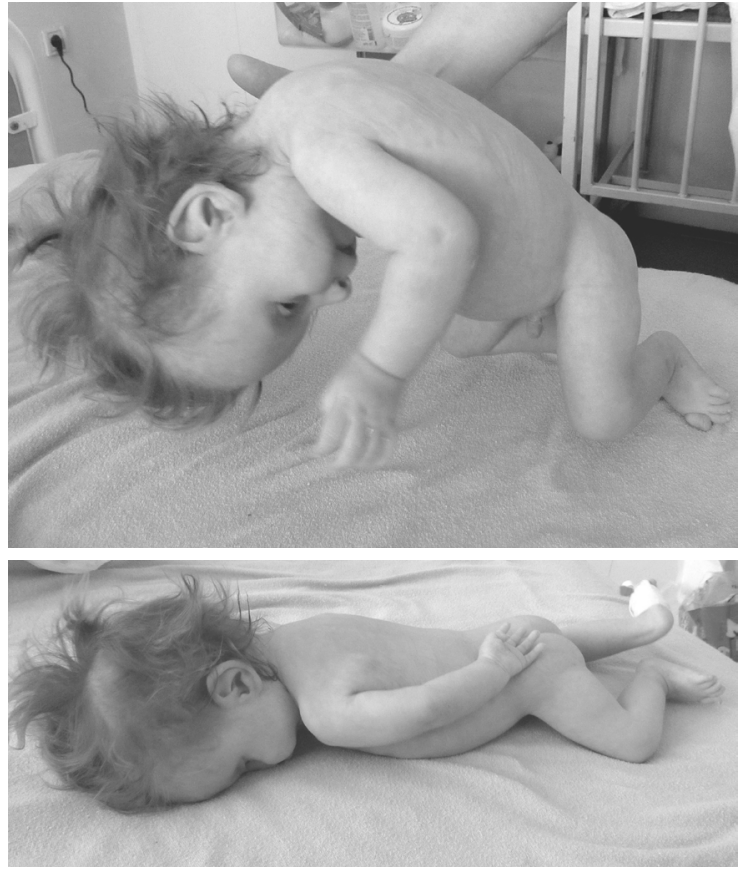

Fig. $5 a$ and $5 b$. Hypotonia and the inability to raise the head in prone position.

of the left kidney. The infant also had two episodes of bronchopneumonia accompanied by high leucocyte count and elevated inflammatory parameters. Klebsiella pneumonia was isolated from nasopharyngeal aspirate. Chest X-ray showed an enlarged heart with prominent pulmonary vasculature as well as 13 ribs and 13 thoracic vertebrae (Fig. 7). Atrial septal defect had been confirmed by echocardiogram after birth. Clinical features described in our patient have been summarized in Table I.

Feeding was difficult in the beginning, adapted infant formula was given by means

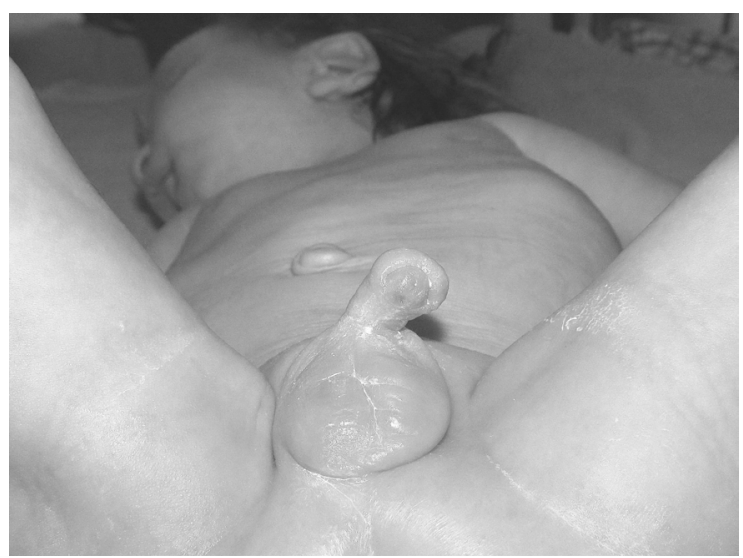

Fig. 6. Hypospadias in our patient. 


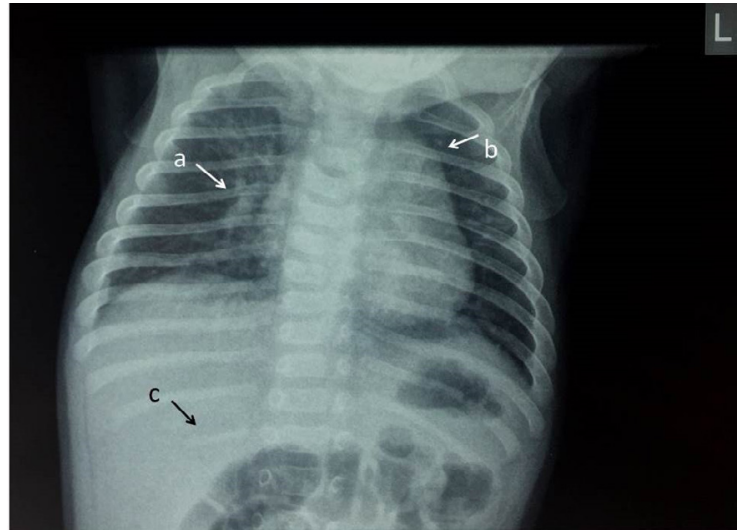

Fig. 7. Chest X-ray: (a) prominent pulmonary vasculature (b) enlarged heart (c) 13 ribs.

of nasogastric tube. Bottle feeding was possible after 2 months of life.

\section{Discussion}

Trisomy 9 mosaicism is certainly rare, but no one knows exactly how often it occurs. The first neonate was reported in 1973.2 In the following four decades there have been sporadic reports of children with trisomy 9 mosaicism associated with variable levels of somatic mosaicism. ${ }^{3}$ The cases that have been described in medical literature are just the tip of an iceberg. This is because the effects of this chromosome disorder can vary very much between individuals and some people may be so mildly affected that they are never diagnosed. The phenotypic appearance may result either from a varying degree of mosaicism or from the different tissues involved. Multiple associated anomalies of central nervous system, face, skull, limbs, heart and urinary tract are described. ${ }^{4-6}$ Most have developmental delay but a few do not. ${ }^{7}$

Delivery is usually slightly before term, but as early as 30 week has also been described. ${ }^{7}$ Intrauterine growth retardation is always present and newborns are small for dates as we identified in our patient. ${ }^{4,5}$ Babies mostly remain short, weight gain is usually slow and children have significant growth retardation. ${ }^{8}$ Head and facial abnormalities as observed in our patient are common in trisomy 9 mosaicism including microcephaly, high and narrow forehead, deep

Table I. Clinical Features of Trisomy 9 Mosaicism Reported by Jones et $\mathrm{al}^{5}$ and Clinical Features Described in Our Patient.

\begin{tabular}{llc}
\hline Physical characteristics & Jones et al. (2013) & Our patient \\
\hline Prenatal onset of growth deficiency & "High incidence" & + \\
Narrow bifrontal diameter & "High incidence" & + \\
Upslanting, short palpebral fissures & "High incidence" & + \\
Deeply set eyes & "High incidence" & + \\
Prominent nasal bridge & "High incidence" & + \\
Micrognathia & "High incidence" & + \\
Low set and misshapen ears & "High incidence" & + \\
Microphthalmia & "Low incidence" & - \\
Facial asymmetry & "Low incidence" & + \\
Cleft lip and palate & "Low incidence" & - \\
Single palmar crease & "Low incidence" & + \\
Joint anomalies & "High incidence" & + \\
Other medical conditions & & + \\
Congenital heart defect & In $2 / 3$ cases & + \\
Dilation of fourth ventricle, hydrocephalus & "Low incidence" & - \\
Absence of optic tract & "Low incidence" & + \\
Hearing loss & "Low incidence" & + \\
13 ribs and thoracic vertebrae & "Low incidence" & + \\
Genitourinary anomalies & "Low incidence" & + \\
\hline
\end{tabular}


set eyes, microphthalmus, bulbous nose, micrognathia and large fontanelle. ${ }^{4,5,8,9}$ Ear abnormalities are seen in more than $90 \%$ of such patients, but hemi facial hypoplasia with facial asymmetry seen in our patient is uncommon and may be related to mosaicism as have been reported by de Ravel et al. ${ }^{10}$ Gazzola et al. ${ }^{7}$ also have described a patient with asymmetrical face. Although many children with trisomy 9 mosaicism and a heart defect have been described in published literature (mostly atrial or ventricular septal defect, persistent ductus arteriosus, rarely Fallot's tetralogy or hypoplastic left heart syndrome), only $10-12 \%$ had a defect that needed surgical correction. ${ }^{8,11}$ Minor anomalies of the genital and reproductive tract are common in boys with trisomy 9 and trisomy 9 mosaicism, particularly hypospadias and undescended testes, as we have seen in our patient. $9,12,13$ Some children with trisomy 9 mosaicism have different brain abnormalities such as excess in cerebrospinal fluid and hydrocephalus, as we have described in our patient. Less frequently, in children with trisomy 9 mosaicism, DandyWalker syndrome-a rare congenital anomaly involving the cerebellum, has been reported. A thinned or even missing corpus calosum is also a rare finding. ${ }^{1,4}$ Different varieties of unusual features of kidney, hydronephrosis, double ureter or vesico ureteral reflux were described. Although urinary tract problems occur in only a minority of children, we found hydronephrosis of left kidney in our patient. 3,11 Seizures are not common in children with trisomy 9 mosaicism, however, no obvious link between structural anomalies of the brain and seizures have been described. ${ }^{13}, 14$ Some authors have reported epilepsy in a patient with trisomy 9 mosaicism. ${ }^{15}$ Although Bruns et $a .^{4}$ reported a high percentage of apnea in children with trisomy 9 mosaicism (20\%), our patient did not have apnea, but he had two episodes of bronchopneumonia. Respiratory tract infections are relatively common in infants with chromosome disorders, mostly caused or aggravated by aspiration of regurgitated feeds. In some children with trisomy 9 mosaicism abnormal lung segmentation has been described without confirmation that this adds to the problem of respiratory tract infections. ${ }^{13,16}$ Inflammatory bowel disease, as well as incorrect development of digestive tract could be seen in children with this chromosome abnormality. ${ }^{13,17}$ Bruns et al. ${ }^{4}$ reported pyloric stenosis and intestinal malrotation in trisomy 9 mosaicism. The literature describes different degrees of mental delay in trisomy 9 mosaicism. ${ }^{8}$ In our patient hypotonia and poor development of motor function was clearly expressed. According to the Denver Developmental Screening test, the patient's gross motor skills, fine motoradaptive, language and personal-social skills in 6 months remain at the level of age $0-1$ month.

There are some clinical and phenotypic similarities between trisomy 9 mosaicism and duplication of $9 \mathrm{p}$ : growth and mental deficiency, microcephaly, deep-set eyes and prominent nose. In partial duplication of $9 p$, the $9 \mathrm{p} 22$ region is mostly responsible for the observed phenotype. The main abnormality of this syndrome is distal phalangeal hypoplasia, which could be seen in trisomy 9 mosaicism as an occasional abnormality. Occasional abnormalities in duplication of $9 p$ are similar to trisomy 9 mosaicism include micrognathia, hydrocephalus, congenital heart defects and genitourinary anomalies. ${ }^{5}$

In conclusion, trisomy 9 mosaicism is a rare chromosomal abnormality with significant clinical variability as a result of a variable degree of mosaicism and differences in the location of tissue involved. Each case report will be a contribution to a better enlightening of the clinical picture, especially with a longterm follow-up.

\section{Acknowledgement}

The authors of this manuscript would like to thank the mother of the child.

\section{REFERENCES}

1. Feingold M, Atkins L. A case of trisomy 9. J Med Genet 1973; 10: 184-187.

2. Haslam RH, Broske SP, Moore CM, Thomas GH, Neillal A. Trisomy 9 mosaicism with multiple congenital anomalies. J Med Genet 1973; 10: 180-184.

3. Bruns D. Presenting physical characteristics, medical conditions, and developmental status of long-term survivors with trisomy 9 mosaicism. Am J Med Genet A 2011; 155A: 1033-1039.

4. Bruns DA, Campbell E. Twenty-five additional cases of trisomy 9 mosaic: Birth information, medical conditions, and developmental status. Am J Med Genet Part A 2015; 167A: 997-1007. 
5. Jones KL, Jones MC, Del Campo M. Trisomy 9 mosaicism. Smith's recognizable patterns of human malformation, 7e. Philadelphia: Elsevier Saunders, 2013: 28-29; 48-50.

6. Kannan TP, Hemlatha S, Ankathil R, Zilfalil BA. Clinical manifestations in trisomy 9. Indian J Pediatr 2009; 76: 745-746.

7. Zen PR, Rosa RF, Rosa RC, Graziadio C, Paskulin GA. New report of two patients with mosaic trisomy 9 presenting unusual features and longer survival. Sao Paulo Med J 2011; 129: 428-432.

8. Stoll C, Chognot D, Half A. Luckel JC. Trisomy 9 mosaicism in two girls with multiple congenital malformations and mental retardation. J Med Genet 1993; 30: 433-435.

9. Miryounesi M, Dianatpour M, Shadmani Z, GhafouriFard S. Report of a case with trisomy 9 mosaicism. Iran J Med Sci 2016; 41: 249-252.

10. de Ravel TJ, Legius E, Brems H, Van Hoestenberghe R, Gillis PH, Fryns JP. Hemifacial microsomia in two patients further supporting chromosomal mosaicism as a causative factor. Clin Dysmorphol 2001; 10: 263267.
11. Patil SJ, Ponnala R, Shah S, Dalal A. Mosaic trisomy 9 presenting with congenital heart disease, facial dysmorphism and pigmentary skin lesions: Intricate issues of genetic counseling. Indian J Pediatr 2012; 79: 806-809.

12. Arnold GL, Kirby RS, Stern TP, Sawyer JR. Trisomy 9: Review and report of two new cases. Am J Med Genet 1995; 56: 252-257.

13. Wooldridge J, Zunich J. Trisomy 9 syndrome: Report of a case with Crohn disease and review of the literature. Am J Med Gen 1995; 56: 258-264.

14. Tarani L, Colloridi F, Raguso G, et al. Trisomy 9 mosaicism syndrome. A case report and review of the literature. Ann Genet 1994; 37: 14-20.

15. Incecik F, Herguner MO, Mert G, Altunbasak S. Trisomy 9 mosaicism presenting with epilepsy, and facial dysmorphism. A case report. Cukurova Medical Journal 2014; 39: 343-346.

16. Saura R, Traore W, Taine L, et al. Prenatal diagnosis of trisomy 9. six cases and a review of the literature. Prenat Diagn 1995; 15: 609-614.

17. Levy I, Levy Y, Mammon Z, Nitzan M, Steinherz R. Gastrointestinal abnormalities in the syndrome of mosaic trisomy 9. J Med Gen 1989; 26: 280-281. 